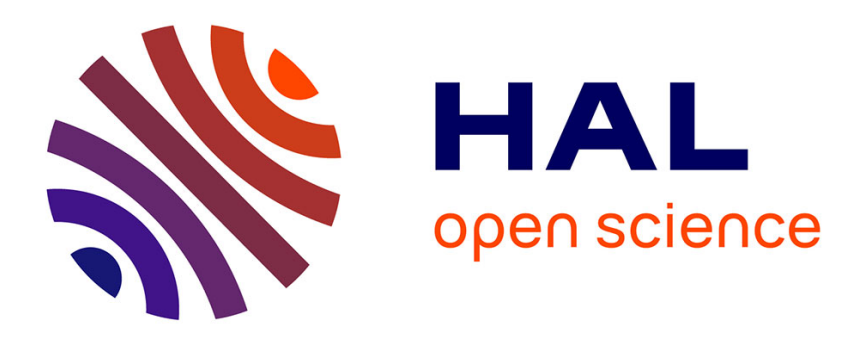

\title{
Slow relaxation and solvent effects in the collapse of a polymer
}

\author{
Thomas Frisch, Alberto Verga
}

\section{To cite this version:}

Thomas Frisch, Alberto Verga. Slow relaxation and solvent effects in the collapse of a polymer. Physical Review E: Statistical, Nonlinear, and Soft Matter Physics, 2002, 66, n 4 part 1, pp.Art. 41807. 10.1103/PhysRevE.66.041807 . hal-00092020

\section{HAL Id: hal-00092020 https://hal.science/hal-00092020}

Submitted on 7 Sep 2006

HAL is a multi-disciplinary open access archive for the deposit and dissemination of scientific research documents, whether they are published or not. The documents may come from teaching and research institutions in France or abroad, or from public or private research centers.
L'archive ouverte pluridisciplinaire HAL, est destinée au dépôt et à la diffusion de documents scientifiques de niveau recherche, publiés ou non, émanant des établissements d'enseignement et de recherche français ou étrangers, des laboratoires publics ou privés. 


\title{
Slow relaxation and solvent effects in the collapse of a polymer
}

\author{
Thomas Frisch* and Alberto Verga ${ }^{\dagger}$ \\ Institut de Recherche sur les Phénomènes Hors Équilibre, \\ UMR 6594, CNRS, Université d'Aix-Marseille, 49, \\ rue F. Joliot-Curie, BP 146, 13384 Marseille, France
}

(Dated: June 4, 2002)

\begin{abstract}
Using molecular dynamic simulations we study the quench of a homopolymer chain into a poor solvent at finite temperature. We show that, depending on the quench depth, there are different relaxation pathways to the collapsed state. Solvent effects are introduced through an effective Lennard-Jones potential depending on the local monomer density. The various relaxation regimes are characterized by the contact correlation function. As the quench depth increases, the system evolves towards a glassy state, and the relaxation dynamics continuously changes from an exponential to a stretched exponential law. The characteristic relaxation time diverges at low temperature following an Arrhenius law, like in the case of strong glasses. We found that the stretching exponent depends on aging in a non-universal way. The solvent modifies the globular state by diminishing the effects of frustration and glassy behavior.
\end{abstract}

PACS numbers: 61.41.+e,87.15.Aa,36.20.Ey

\section{INTRODUCTION}

When an unfolded polymer chain is quenched into a poor solvent it collapses to form a compact globule. This phenomenon has received in recent years a lot of attention in the literature from both theoretical and experimental points of view, due mainly to its application in protein folding and in basic polymer science [1-9]. However, the physical characterization of the relaxation dynamics as a function of the quench depth and the slow evolution of the globular metastable states still remain to be thoroughly investigated [10]. Moreover, experimental results on single polymer chains in the globular state are still scarce because of the difficulty in avoiding aggregation $[3,4,11]$. Indeed, it was recently shown that the homopolymer globular state shares many properties with amorphous materials like glasses [12-14]. Relaxation becomes exceedingly slow at low temperatures and aging effects appear, in practice glassy systems may never reach equilibrium on laboratory times scales $[15,16]$. The glassy behavior of a homopolymer quenched below the $\Theta-$ point was recently addressed numerically in [14], where it was shown that a close resemblance exists between the correlation or structure functions measured in glasses and in single polymer chains. The usual picture of polymer collapse when quenched below the $\Theta$-point $[9,17-24]$, shows that non-equilibrium effects are important, especially the influence of the solvent, the relaxation pathways and the glassy properties of the globular state.

The effect of a solvent on the polymer collapse pathways was recently studied numerically $[10,25,26]$. Simple Langevin dynamics and molecular dynamics including Lennard-Jones solvent molecules were compared [10].

\footnotetext{
*Electronic address: frisch@irphe.univ-mrs.fr
}

${ }^{\dagger}$ Electronic address: verga@irphe.univ-mrs.fr
It was shown that the main differences between Langevin dynamics and molecular with solvent is that in this last case the polymer does not get trapped in local minima as observed with Langevin dynamics. Although the evolution to the globular state is similar in both cases, the effect of frustration is greatly diminished in the presence of solvent. The main effect of the solvent is then to reduce the attractive interactions between monomers inside the globular state, facilitating the escape from local energy minima.

We address the question of the quench depth influence on the relaxation dynamics using molecular dynamics in a canonical ensemble and we introduce a simplified description of the polymer interactions with the solvent. We use an effective many particle interaction (at variance to the usual binary Lennard-Jones potential) through a local density dependent potential: the interaction between two separated monomers depends on the number of their neighbors. Our simplified solvent model neglects the hydrodynamic effects (long range interactions). This approach is somewhat different to usual models of hydrophobic interactions based on the accessible surface area (see for instance Ref. [27] where a many-body interaction was proposed to describe the hydrophobic effect in protein folding). We find that the qualitative features of the chain-globule transition depend on the quench depth. For small values of the temperature difference $\Delta T=|T-\Theta|$, below the $\Theta$-point, the polymer collapses isotropically. For larger values of $\Delta T$, initial density fluctuations grow to form clusters (also referred as to pearls $[19,20,28]$ ), or, at higher values of $|\Delta T|$, to form sausage like structures as predicted by de Gennes [2]. For large values of $|\Delta T|$ solvent effects are neglected the globular density may be non-uniform, the topological chain constraint imposing frustration. We observe that these metastable states tend to disappear in the presence of solvent interactions. The polymer does not get trapped in high energy metastable states (high energy 
minima) even if the quench amplitude is very strong [10]. These results are presented in section $\S$ II together with the numerical scheme and the solvent model.

The description of the glassy behavior of the globular state is presented in section $\S$ III. Previous studies of this problem where done using hard sphere potentials [14]. In order to investigate quantitatively the glassy dynamics we must take into account the fact that a polymer, in contrast to usual glasses, is formed by a chain of monomers and not of free molecules. It is then desirable to introduce specific quantities that incorporates this topological constraint. We study the relaxation dynamics by means of a contact correlation function. This function characterizes the surroundings of a given monomer and the duration of the contact with its neighbors. A related quantity, the cage correlation function, was introduced by Rabani et al. for Lennard-Jones systems [29]. We show that as one varies the depth of the quench below the $\Theta$-point the monomer-monomer contact correlation function goes from a classical exponential decay to a stretched exponential decay. The stretched exponential relaxation is characterized by two parameters, a relaxation time $t_{c}$ and an exponent $\beta$. We found that these parameters depend not only on the temperature but also on the system age. These aging effects appear to be very complex and escape a simple description in terms of universal power laws. Finally, we discuss the effects of the solvent on the polymer relaxation. We show that, in the presence of a solvent, the exponent $\beta$ approaches one, even for low temperatures, and aging effects almost disappear. Conclusions are drawn in section IV.

\section{COLLAPSE PATHWAYS AND SOLVENT MODEL}

\section{A. The numerical method}

The numerical simulations were performed using the Nosé-Hoover algorithm of molecular dynamics in the canonical ensemble (with fixed temperature and fluctuating energy) [28, 30, 31]. The polymer chain contains $N$ monomers interacting through a classical long-range van der Waals forces. The connectivity of the chain was implemented by using a strong anharmonic potential for neighboring molecules. Most of the results presented here were obtained with $N=400$. The Nosé-Hoover equations for the $i$ monomer are

$$
\begin{aligned}
\dot{\mathbf{r}_{i}} & =\mathbf{p}_{i} / m_{i}, \\
\dot{\mathbf{p}_{i}} & =-\frac{\partial V_{i}}{\partial \mathbf{r}_{i}}-\xi \mathbf{p}_{i}, \\
\tau_{\xi}^{2} \dot{\xi} & =\frac{2 K}{k_{B} T}-(3 N-6),
\end{aligned}
$$

where $\mathbf{r}_{\mathbf{i}}=\left(x_{i}, y_{i}, z_{i}\right)$ and $\mathbf{p}_{i}$ are the position and momentum; over dots represent time derivatives. In order to control the mean kinetic energy $K=\sum_{i=1}^{N} p_{i}^{2} / 2 m_{i}$ a "friction" variable $\xi$, and a characteristic time of the heat bath exchanges $\tau_{\xi}=2 Q / k_{B} T$ are introduced $(Q=$ const. $=0.1)$. The total number of degrees of freedom is $3 N-6$ since we subtracted the translation and rotation of the center of mass.

The potential $V_{i}=\sum_{j=1}^{N} V\left(r_{i j}\right)$ is the interaction energy of monomer $i$ with all other monomers $\left(r_{i j}\right.$ being the distance between monomers $i$ and $j$, we always assume $i \neq j$ ). It has two contributions $V=V_{1}+V_{2}$ : (i) the valence interaction between two neighboring monomers

$$
V_{1}\left(r_{i j}\right)=a\left(r_{i j}-d_{0}\right)^{2}+b\left(r_{i j}-d_{0}\right)^{4}, \quad \text { for } i=j \pm 1,
$$

where $d_{0}=1$ is the equilibrium distance (used as the length unit) and $a=30, b=100$ are constants characterizing the harmonic and anharmonic interactions (measured in energy units to be specified below); (ii) the Lennard-Jones interaction between non-neighboring monomers

$$
V_{2}\left(r_{i j}\right)=\eta\left[\left(\frac{\sigma}{r_{i j}}\right)^{12}-\left(\frac{\sigma}{r_{i j}}\right)^{6}\right], \quad \text { for } i \neq j, j \pm 1,
$$

where $\eta=1.0$ is the potential depth (chosen as the unit of energy) and $\sigma=1.0$ the van der Waals radius (note that we chose $d_{0}=\sigma$ but neighboring monomers $i=j \pm 1$ do not interact with $V_{2}$ ). In the numerical computations a cut-off of the long range attractive force at $r_{c}=2.5 \sigma$ was used in order to introduce the Verlet list algorithm to efficiently compute the force term in (2). To compute the Verlet list we defined two characteristic distances, an inner radius $r=r_{c}$ and an outer one $r=r_{v}=2.7 \sigma$ [30, 31]. Time stepping was performed using an implicit Verlet-Newton-Raphson method [31]. The time step was fixed at $\Delta t=0.210^{-3}$. We used $1000 \Delta t$ as the unit of time. The initial state consists in a self-avoiding walk generated by a Monte-Carlo method. This state is appropriated as an initial condition for a polymer above the $\Theta$-point (see below Fig. 1a).

The $\Theta$-point was found at the temperature $\Theta \approx 0.45$, for the above set of numerical parameters. This value was obtained by two equivalent methods, using the gyration radius and the specific heat as functions of the temperature. A solid state transition, also identified with the specific heat, is located about $T_{s}=0.1$. In the numerical simulations we explored the temperature range $T=0.05-0.35$, which lies below the $\Theta$-point.

\section{B. The solvent model}

Fully explicit simulations with solvent particles are still very expensive numerically in three dimensions [10, 25]. However, as recently shown by Chang and Yethiraj [10], the numerical simulations of a polymer in a solvent reveal that the collapse pathways change when polymer-solvent interactions are taken into account. They used LennardJones potentials to describe solvent-solvent and solventpolymer interactions. In the case of a strong quench, 
they observed that the polymer collapses by forming first a sausage like structure, but instead of getting trapped in high potential energy states, it slowly evolves towards an isotropic distribution. The main effect of the solvent is to enhance the mobility of monomers inside the globule by an effective reduction of the attractive interactions, and simultaneously to increase the attraction between monomers exposed to the solvent. This is the usual "hydrophobic effect" that was also modeled in terms of the "accessible surface area," or similarly in terms of the local density of monomers using a non-additive attractive potential [27]. The interest of these methods is their reduced computational cost, since the solvent degrees of freedom are not explicitly computed, but the solvent action is modeled by hydrophobic interactions. However, the potential used is phenomenological and its physical origin remains to be investigated.

In order to describe the hydrophobic effect we propose a method based on the idea that the van der Waals interactions (long range attraction) are modified by the presence of the solvent, or equivalently by the number of neighbors of a given monomer. We assume that the attractive interactions between monomers are modulated by their local density (proportional to the number of neighbors). This assumption is compatible with a virial development in powers of the polymer density, and with the effective solvent-mediated interaction measured by Chang and Yethiraj [10]. The form of the interaction remains of the Lennard-Jones form,

$$
V_{2}\left(r_{i j}\right)=\eta\left[\left(\frac{\sigma}{r_{i j}}\right)^{12}-c_{i j}\left(\frac{\sigma}{r_{i j}}\right)^{6}\right]
$$

but its intensity is a functional of the number of monomers surrounding a given one,

$$
c_{i j}=1-\lambda\left(n_{i}+n_{j}-4\right) / 2,
$$

where $n_{i}$ is the number of monomers within a sphere of radius $r_{v}$ around monomer $i$ (as given by the Verlet list in our algorithm), and $c_{i j}$ is proportional to the mean number of neighbors of the $(i, j)$ pair (excluding the near neighbors, as indicated by the term -4 ). The coefficient $\lambda$ measures the strength of the hydrophobic effect, and is chosen small enough to ensure that $0<c_{i j}<1$ even in the high density state (with $\lambda=0.01$ ). As the number of monomers surrounding the two monomers increases, $c_{i j}$ decreases and $V_{2}(r)$ becomes less attractive. As a result, inside the globule, where the numbers $n_{i}$ are large, monomer interactions becomes only slightly attractive allowing for a high monomer mobility. In contrast, the surface monomers would have a smaller value of $n_{i}$, thus a larger $c_{i j}$, and stronger attractive interactions. Extensions of this model may be considered in order to account for example, for a concentration dependent FloryHuggins parameter $\chi$, or for more complex effective potential forms (multiple minima and energy barriers between stable states).

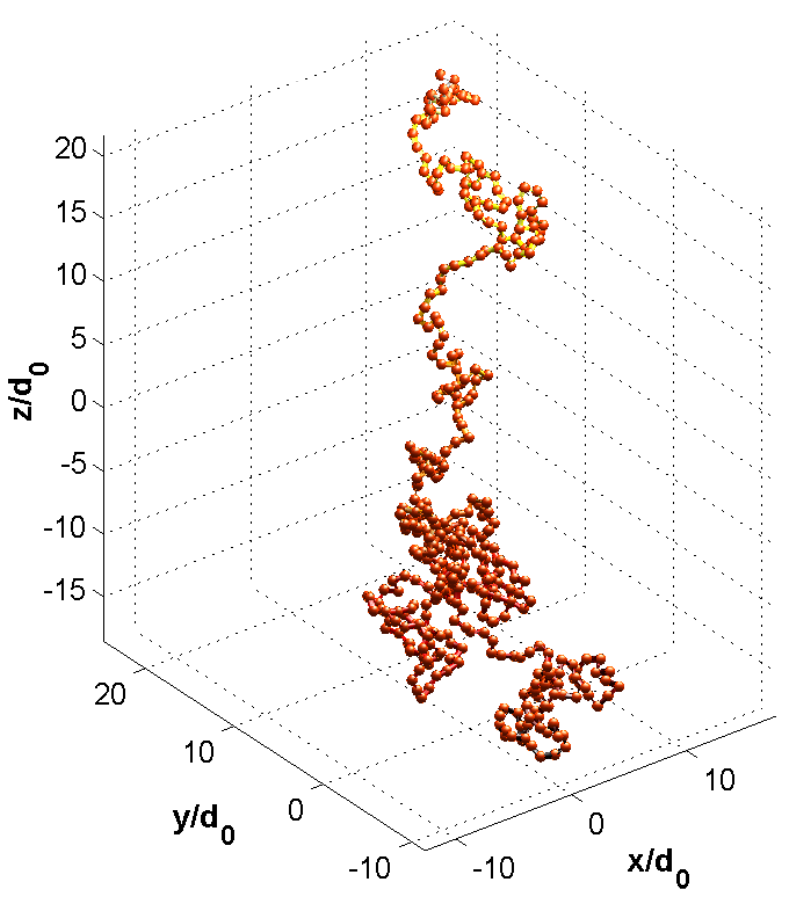

(a)

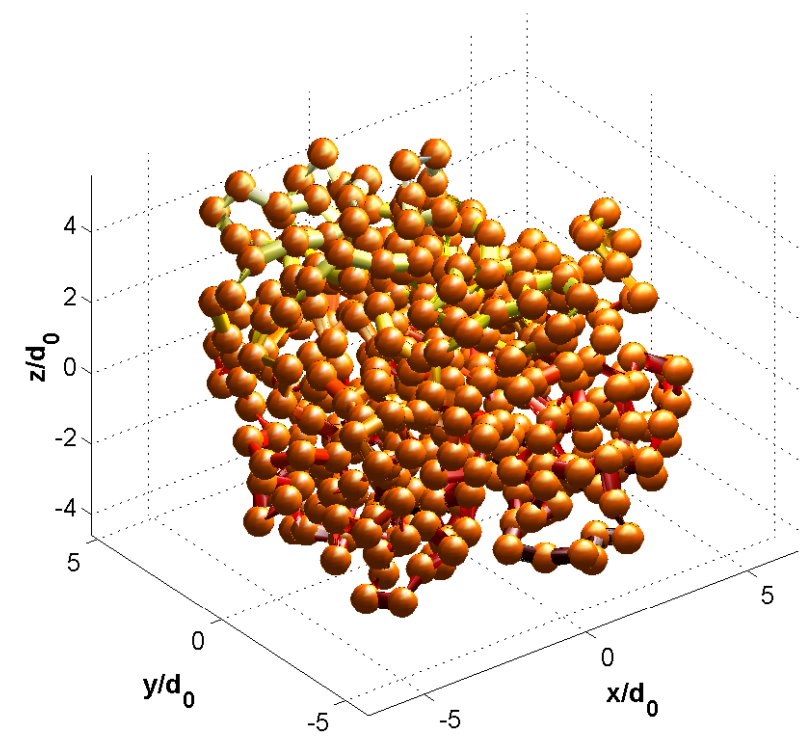

FIG. 1: Molecular dynamic simulation of a $N=400$ homopolymer for $T=0.35$. (a) A self-avoiding random walk generated by a Monte-Carlo method $(t=0)$; (b) the final isotropic globular state $t=150$.

\section{The collapse pathways}

Experimental observation of the collapse (coil to globule transition) in synthetic polymers is difficult because at concentrations accessible to experiments, the intramolecular collapse of single chains competes with the intermolecular aggregation of chains. Consequently there has been only a few studies on the kinetics of the collapse transition. These studies are too scarce to con- 


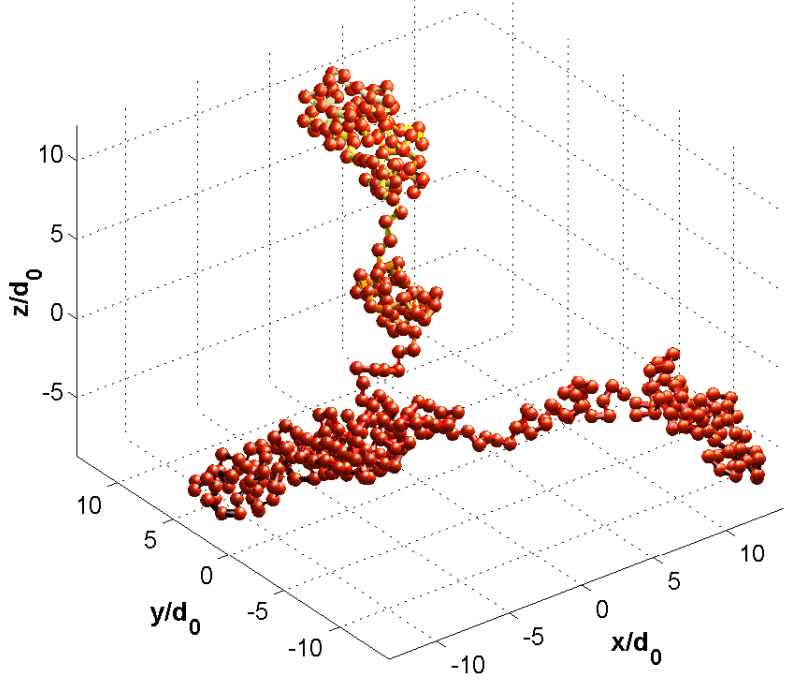

(a)

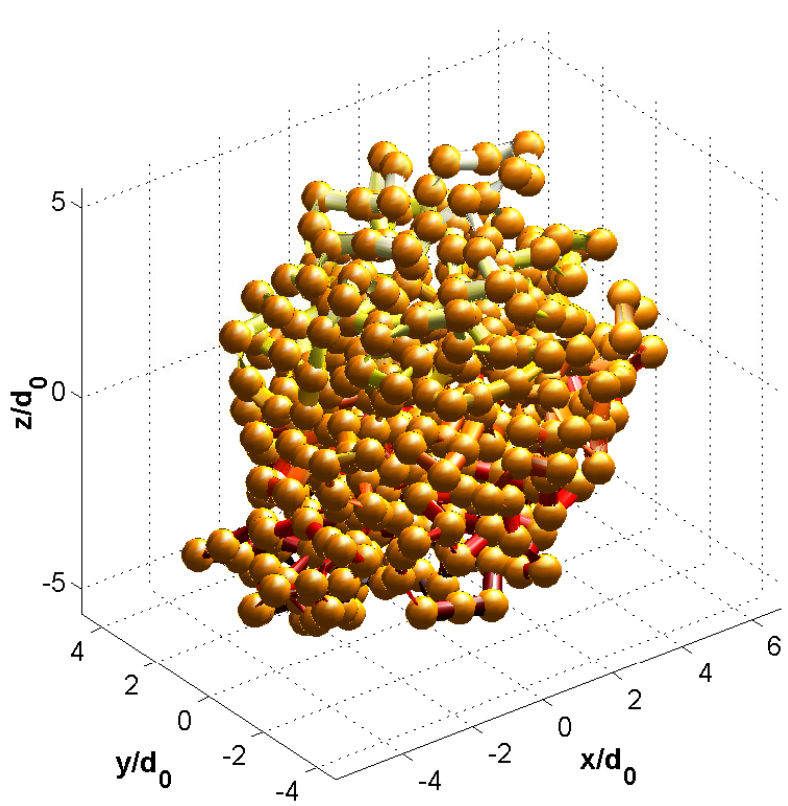

(b)

FIG. 2: Molecular dynamic simulation of a $N=400$ homopolymer for $T=0.25$. (a) The pearls regime $t=10$; (b) globule $t=100$.

firm or infirm the possible theoretical pictures predicted for the collapse. However, the main picture that has emerged from different numerical simulations is that collapse proceed following a series of steps, first the rapid formation of blobs (also referred as pearls) that grow and then merge, then the formation of a globular state [19]. In [10] the importance of the quench depth on the collapse was also underlined, and it was noted that there exists a low temperature regime (strong quench) for which the sausage-like structures can be observed after a rapid transitory stage of blob formation.

We study the collapse pathways as a function of the quench depth $\Delta T$, for the polymer without solvent (5) and also for the polymer in a solvent (6). We start the
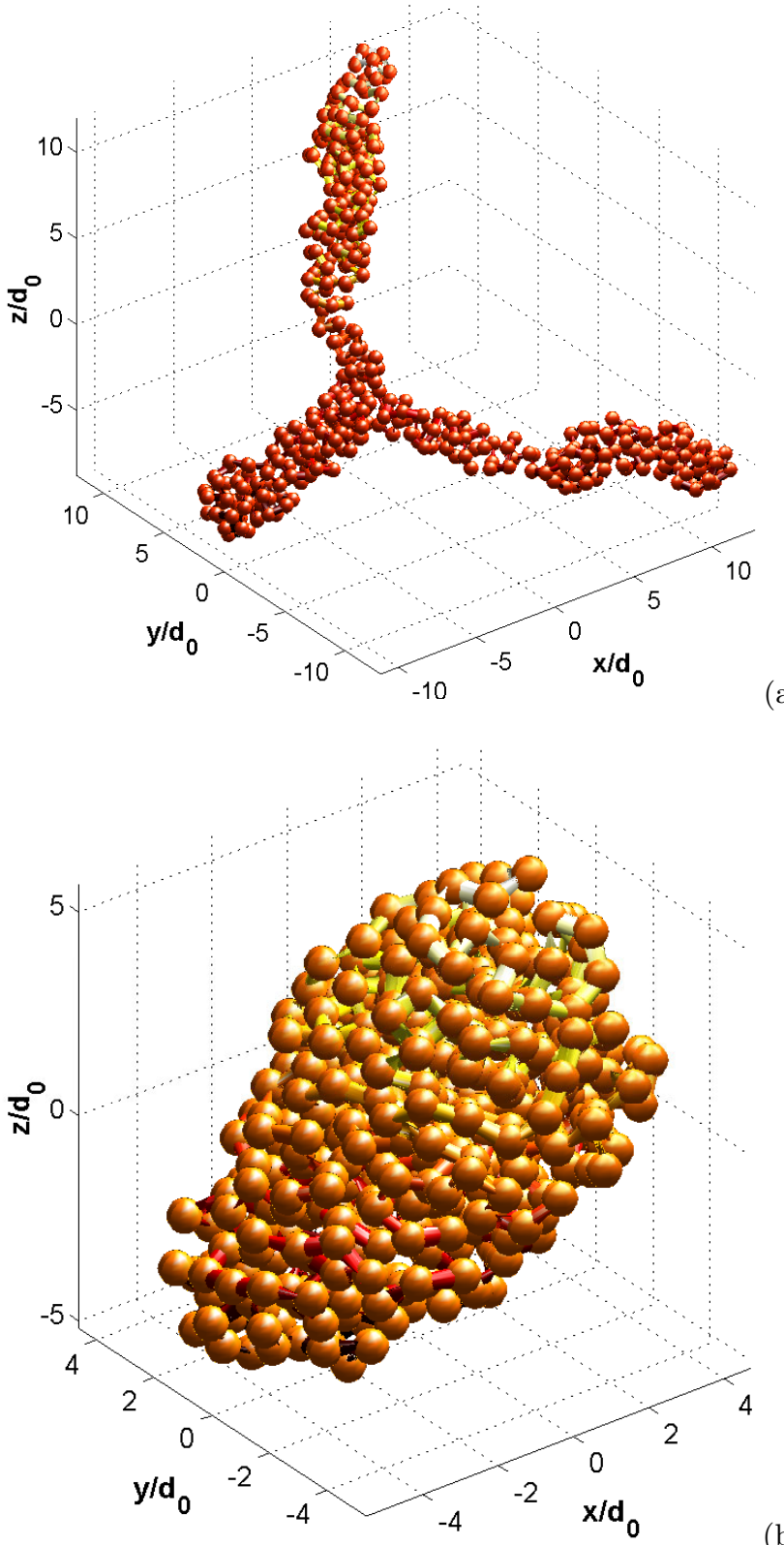

(b)

FIG. 3: Molecular dynamic simulation of a $N=400$ homopolymer for $T=0.05$. (a) Sausage regime $t=20$; (b) non spherical globule trapped in a high potential energy metastable state $t=200$.

simulation from an initial polymer in the coil state obtained using a self-avoiding random walk generated above the $\Theta$-point (Fig. 1a). We consider first the case of small quench below the $\Theta$-point $\Delta T=0.1$ corresponding to $T=0.35$. In this regime the mobility of each monomer is quite high and monomer position fluctuations are so large that density correlations disappear. The polymer collapses towards a homogeneous final state in the form of a spherical globule (Fig. 1b). The collapse evolution could in principle be described by a simple mean field 
theory [1].

We then decrease the temperature (increase the quench depth) to $\Delta T=0.2, T=0.25$. In this regime the attractive interactions are sufficiently strong so that small clusters of monomers forming from initial density fluctuations (pearls) can survive and grow (Fig. 2a). This regime is thus characterized by the formation of pearls that have been first observed in numerical simulations $[19,20,22,23]$ and described theoretically in [17] and [18]. The numerical results have shown that the collapse is largely dominated by the growth of pearls. Pearls nucleate with more ease from the ends of the polymer or from regions of high curvature. During the collapse process, pearls are locally in quasi-equilibrium, linked to one another by a fluctuating stretched chain. The entropic tension exerted on the chain can significantly reduce thermal fluctuations of the linking chain. The behavior of a single pearl under tension has been investigated [11, 28, 32-39], where it was shown that above a critical tension pearls unwind. The equilibrium states attained after successive generations of pearl merging is a well mixed globule whose state is liquid like. The final condensed state (Fig. 2b) consists in a compact globule with a gyration radius scaling as $N^{1 / 3}$.

For a very strong quench, for which the resulting temperature lies below the solidification point $\Delta T=0.4$, $T=0.05$, the kinetic energy of each monomer is so small that the system is dominated by monomer-monomer interactions. As a result, monomers tend to accrete rapidly to their second neighbors which then accrete to their next neighbors triggering a cascade of long-lived attractive interactions. The resulting collapsing object has a sausage like shape [2, 10] (Fig. 3a). In this case, the relaxation dynamics is complex and very slow. The equilibrium states cannot be reached within reasonable time scales. We observed, in the case of long chains $(N>500)$, that different sausages form along the chain linked by more flexible regions. The chain bending around these regions allow the sausages to aggregate in a random way. This process leads to the formation of a compact set of disordered sausages resulting in a highly frustrated state. The quasi-equilibrium attained is an inhomogeneous glassy state sharing some similarities with an amorphous solid (Fig. 3b).

It is important to note out that there is not a well defined temperature transition between this three regimes but rather a smooth transition from the isotropic regime to the sausage regime. Therefore, it is possible to observe a coexistence of sausage and pearls at a given quench depth. As a consequence of the existence of the different regimes described above, each one having its own time scale, and a typical dependence on the thermodynamical variables $N$ and $T[18,40]$, one must rule out a simple description of the collapsing process.

We now focus our attention on the collapse pathway for a polymer in a solvent using the model introduced in (6). We are specifically interested in the effect of the solvent on the sausage regime $\Delta T=0.4, T=0.05$, described

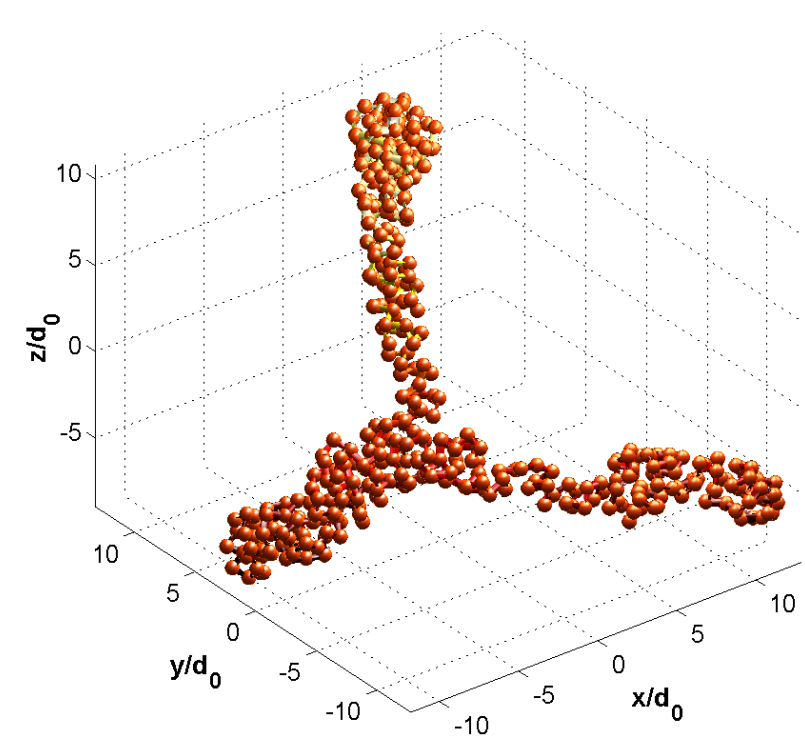

(a)

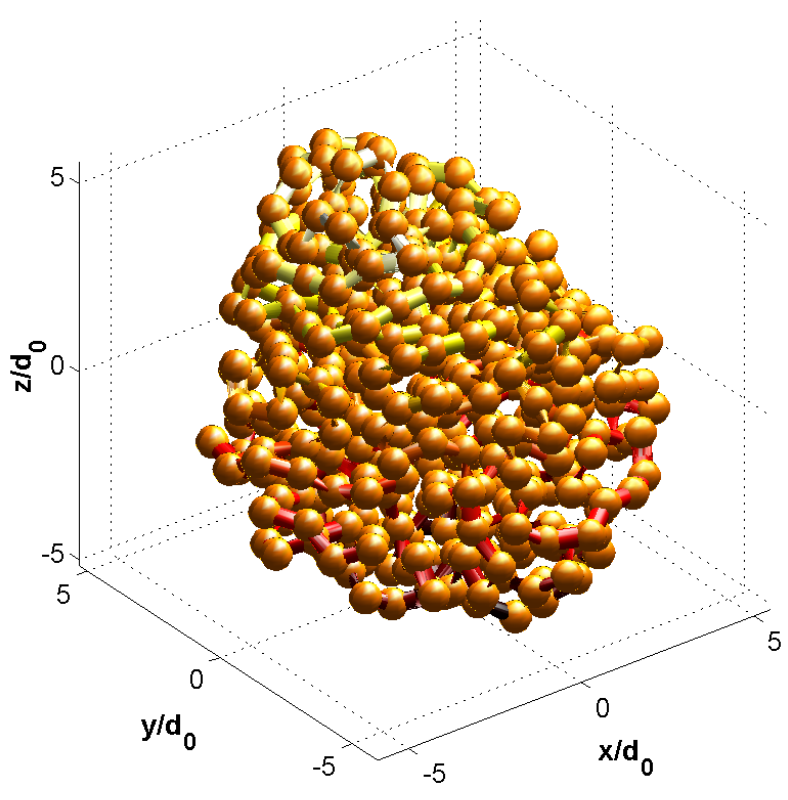

(b)

FIG. 4: Solvent effects in a $N=400$ homopolymer for $T=$ 0.05 , and $\lambda=0.01$. a) the sausage regime $t=20$; (b) the globular state is spherical and liquid-like $t=200$.

just above. Since the effect of the solvent is proportional to the density of the polymer we expect that in the first step of the collapse the solvent has only a very small effect. As a consequence, the second neighbors attraction is dominant and we recover the sausage like structure (4 a). However, as the polymer collapses its density increases so that the attractive forces between monomers slightly decrease, as seen from Eq. (6). The monomers inside the globule do not get trapped in high energy meta-stable states owing to the monomer high mobility. The final state is thus a liquid globule with less monomer mobility on its periphery (Fig. 4b). This effect may also increase the effective surface tension. The final globular state is 


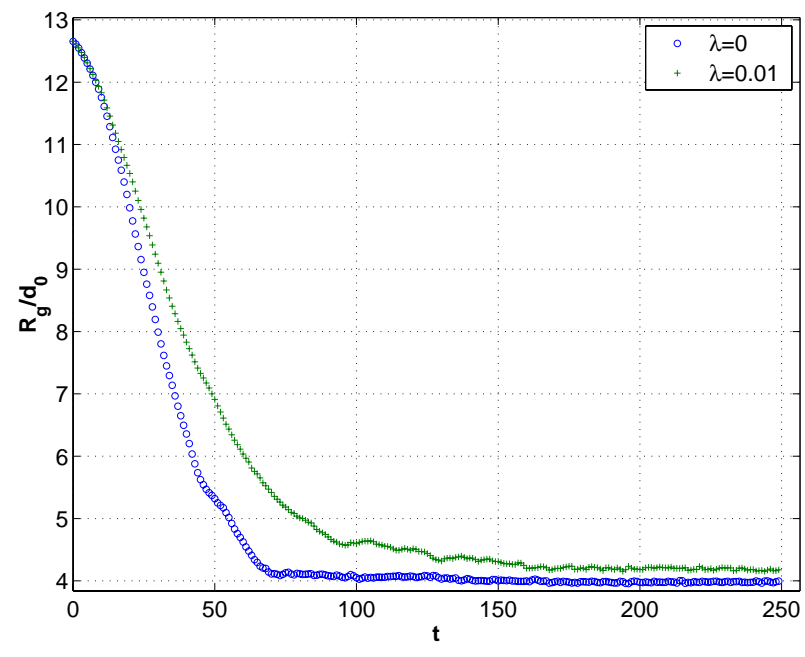

FIG. 5: Gyration radius $R_{g}$ versus time $t$; $\circ$ : without solvent $\lambda=0 ;+$ with solvent $\lambda=0.01$.

rather spherical, in contrast to the case without solvent interactions for which we observed irregular forms.

One possible experimental technique to follow the polymer collapse is to measure the time evolution of the gyration radius by light scattering of a highly dilute solution (in order to get single molecule properties). We compared the evolution of the gyration radius with and without solvent effects (see Fig. 5) for the same temperature $(T=0.05$, strong quench) starting from the same initial conditions.

In absence of solvent effects, the gyration radius diminishes first rapidly until $t=50$ and then decreases irregularly to its quasi-equilibrium value. This function is clearly not smooth revealing the signature of a complex process associated with the aggregation of sausages. It is hard to define a precise value for the collapse time since the ultimate asymptotic value may not be known a priori. Moreover, the functional form of the time dependency of the gyration radius differs with varying temperature, impending to find a simple scaling law. However, a simple examination of Fig. 5, reveals that a "characteristic" collapse time may be defined as for instance the time for which the gyration radius reaches its equilibrium value. In the present case it is of order $t \sim 70$. Other numerical computations show that the collapse time decrease we the raise of the temperature, as long as we remain below the $\Theta$-point.

In presence of solvent effects, the time evolution of the gyration radius is much more smooth, reflecting a lower frustration of compact regions. We observe in Fig. 5, that the collapse time is now of the order of $t \sim 150$, greater than in absence of solvent. The value reached by the gyration radius is larger in presence of solvent interactions than without solvent effects. Indeed, on one hand with solvent, the effective intensity of the attractive forces is diminished. On the other hand, the form of the globule in absence of solvent is not spherical, and the
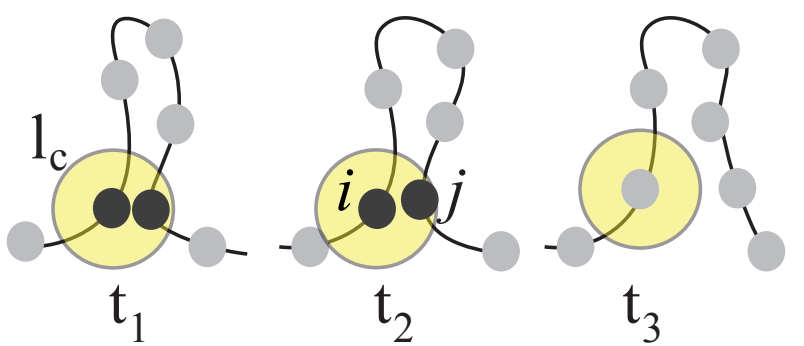

FIG. 6: Sketch of a polymer illustrating the definition of the contact correlation function. Time $t_{1}$, the two monomers (in black) $i$ and $j$ are in contact: both are inside the sphere of radius $l_{c}$ around monomer $i$. Time $t_{2}$, monomers $i$ and $j$ are still in contact. Time $t_{3}$, monomers $i$ and $j$ (now in gray) are no more in contact, the distance between them became larger then $l_{c}$.

actual value of the gyration radius varies significantly with the initial condition and the relaxation pathway.

The observed final state (as shown in Fig. 4b), characterized by a high mobility of monomers and high density, is reminiscent of the molten globular state often found in the context of protein folding.

\section{THE GLASSY STATE}

The globular state dynamics has been studied using different numerical methods [41, 42] (Monte-Carlo), and [43] (molecular dynamics with hard sphere interactions). It has been reported a slowing down of the evolution characteristic of glasses [41, 42], and an order-disorder transition inside the globule [43]. As we increase the quench depth, we also noticed that the dynamics inside the globule slows down and became more complex with a very large relaxation time. Homopolymer transition are classically characterized by the behavior of the meansquared gyration radius $R_{g}$ as a function of the temperature. However, the gyration radius does not provide useful information on the internal structure of the globule and more appropriate quantities need to be introduced. In order to investigate quantitatively the glassy dynamics we must take into account the fact that a polymer, in contrast to usual glasses, is formed by a chain of monomers and not of free molecules. It is then desirable to introduce a specific quantity that incorporates this topological constraint in order to study the relaxation dynamics.

We introduce the contact correlation function $P\left(t_{w}, t\right)$ that characterizes the surroundings of a given monomer and the duration of the contact with its neighbors. It is a function of two variables, the waiting time $t_{w}$ and time $t ; P\left(t_{w}, t\right)$ represents a mean probability of persistence of the contact of two monomers between time $t_{w}$ and $t_{w}+t$. We assume that two monomers are in "contact" if their distance is smaller than the length $l_{c}=2^{1 / 6} \sigma$, the equilibrium point of the van der Waals forces, Fig. 6 . (We checked that our results do not depend on the pre- 
cise value of $l_{c}$.) A related quantity, the cage correlation function, has been introduced by Rabani et al. for Lennard-Jones systems [29] in another context. The contact correlation function can be explicitly written as

$$
P\left(t_{w}, t\right)=\left\langle\frac{1}{\mathrm{nc}\left(t_{w}\right)} \sum_{i, j} \chi_{i, j}\left(t_{w}, t\right)\right\rangle,
$$

with

$$
\chi_{i, j}\left(t_{w}, t\right)=\prod_{k=0}^{[t / \delta t]} H\left[l_{c}-d_{i j}\left(t_{w}+k \delta t\right)\right]
$$

where $H$ is the Heaviside function and $d_{i j}(t)=\mid \mathbf{r}_{i}(t)-$ $\mathbf{r}_{j}(t) \mid$ is the distance between non-neighboring monomers $i$ and $j$. The function nc $\left(t_{w}\right)$ is the number of contacts at time $t_{w}$. The double sum is on all indexes $i$ and $j$ except $i=j$ and $i=j \pm 1$. The sampling time $\delta t$ is chosen small enough, $\delta t=100 \Delta t$ so that our result do not depend on the sampling time. Brackets denote an average over ten independent runs with different initial conditions. (An average over five runs leads already to sensibly the same results.) We finally normalized the contact correlation function to unity by dividing it by the number of contacts $n_{c}\left(t_{w}\right)$ at time $t_{w}$,

$$
\operatorname{nc}\left(t_{w}\right)=\sum_{i, j} \chi_{i, j}\left(t_{w}, 0\right)
$$

thus with this normalization $P\left(t_{w}, 0\right)=1$ for all waiting times. Therefore, the function $P\left(t_{w}, t\right)$ in Eq. (8) measures the mean probability for two non-bonding neighbors in contact at time $t_{w}$ to be still in contact at time $t_{w}+t$ and to have remained in contact between time $t_{w}$ and time $t_{w}+t$. One important property of $P\left(t_{w}, t\right)$ is that it is insensitive to the number of monomers provided that $N$ is large enough, this results from the fact that $P$ is an average over pairs of monomers (we verified this property for the $N=400$ simulations).

\section{A. Numerical results}

The contact correlation function is also a function of the temperature. We have measured its time evolution during the collapse process for a large range of temperatures below the $\Theta$-point. The origin of the waiting time $t_{w}=0$ is taken to correspond to the instant of the quench. We computed $P\left(t_{w}, t\right)$ for a large range of $t_{w}$ and $t$ values with their maximum values chosen longer then the maximum characteristic time of the collapse mentioned in the end of IIC. We observed on one hand for all cases we considered below the $\Theta$-point, a relaxation of $P\left(t_{w}, t\right)$ towards zero as a function of $t$. On the other hand, the $t_{w}$ dependence of $P\left(t, t_{w}\right)$ is very small at high temperature and becomes more important as we lower the temperature. Figure 7 is a surface plot in the $\left(t_{w}, t\right)$

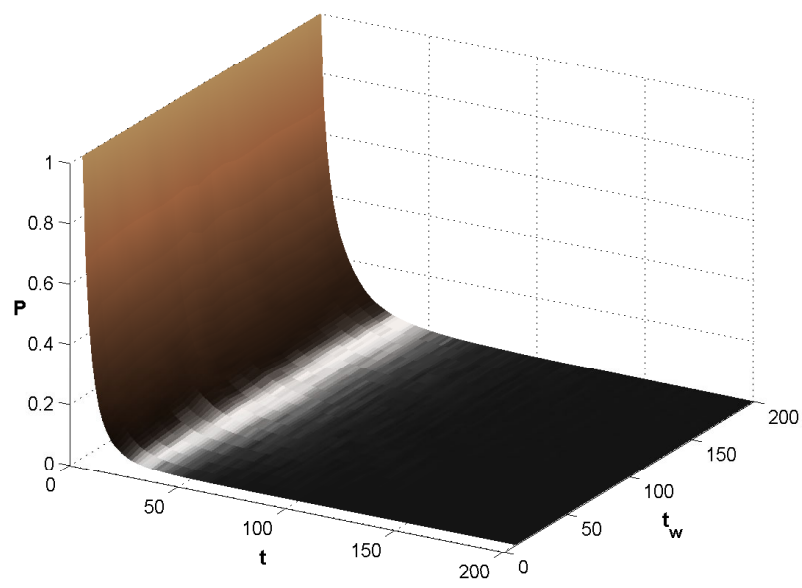

FIG. 7: Contact correlation function $P\left(t_{w}, t\right)$ for $T=0.1$.

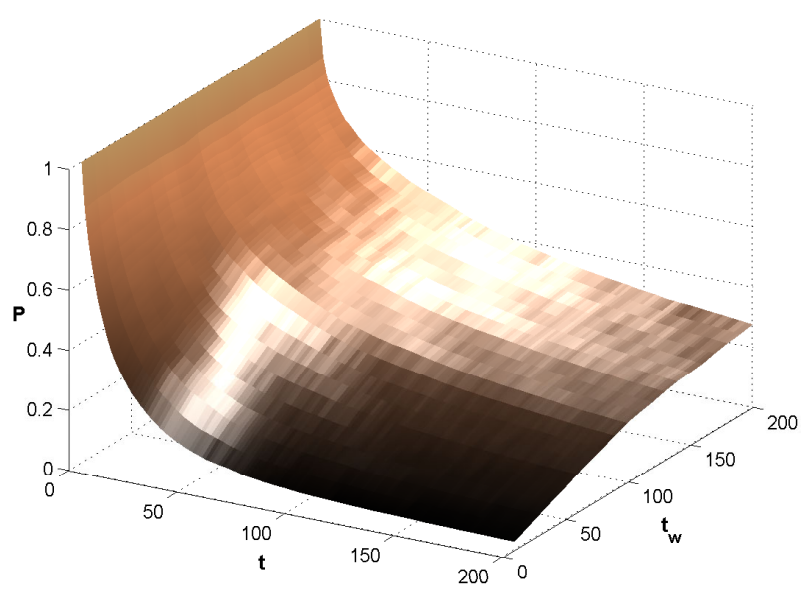

FIG. 8: Contact correlation function $P\left(t_{w}, t\right)$ for $T=0.05$.

space, of the contact correlation function at a temperature $T=0.1$, its $t_{w}$ dependance is quasi-absent and the relaxation is quite smooth. However, at a lower temperature $T=0.05$, as shown in Fig. 8, the relaxation is much slower than at $T=0.1$. Moreover, there is clear $t_{w}$ dependance signaling the onset of aging phenomena.

\section{The long waiting time behavior}

We shall first investigate the long waiting time behavior of the contact correlation function. We observed that for waiting times larger than $t_{w}=200$, the system is in a quasi-equilibrium state, except for low temperatures. Indeed, for low temperatures the system sets in a metastable state and shows glassy behavior. For larger temperatures, the $t_{w}$ dependence of $P\left(t_{w}, t\right)$ vanishes, as long as $t_{w}$ is large enough, and thus $P\left(t_{w}, t\right)$ becomes a function of $t$ which we denote by $P_{e q}(t)$. We were able to obtain quasi-equilibrium states down to temperature $T=0.07$. Below this temperature the dynamic is too slow, so that an equilibrium cannot be attained in a rea- 


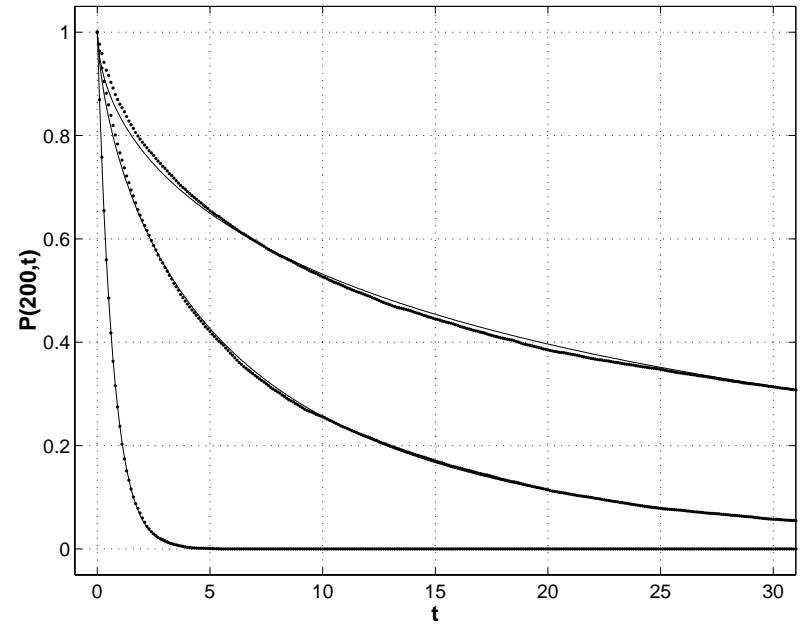

FIG. 9: The equilibrium contact correlation function for three different temperatures: bottom $T=0.3$, middle $T=0.15$ and top $T=0.07$. Data is fitted solid curves) with formula (11), with (from bottom to top) $\beta=1,0.87,0.5$, and $t_{c}=0.7,2$, 23 .

sonable computer time. We show in Fig. 9, the time behavior of the equilibrium correlation function for three different temperatures. Dots on Fig. 9 correspond to raw data which are fitted by a stretched exponential function,

$$
P_{e q}(t)=\exp \left[-\left(\frac{t}{t_{c}(T)}\right)^{\beta(T)}\right]
$$

represented by a continuous line. This function contains two free parameters $t_{c}(T)$, related to the relaxation time, and the stretching exponent $\beta(T)$, which can be precisely determined by an error minimization routine. For a small quench at $T=0.3$, the relaxation is very rapid while for $T=0.15$ and $T=0.07$ the relaxation time is much longer. Fitting these curves allows us to determine the two parameters $\beta(T)$ and $t_{c}(T)$. The data was fitted using the whole range $t=[0,200]$. These two parameters depend in a non-trivial way on the temperature $T$.

The origin of the stretched exponential behavior is at this moment not fully understood since there does not exist yet a simple theory of the glass transition for a polymer. The well known mode coupling theory [44, 45], applicable to supercooled liquids, does not take into account the topological constraint of the polymer chain. This constraint introduces spatial frustration and density inhomogeneities that are neglected by the mode-coupling theory. However, it is worthy adding that stretched exponential evolution laws are now common in condensed matter (glasses, electrical conductivity, random walk in disordered media) and are sometimes referred as KohlrauschWilliams-Watts laws [45]. Stretched exponential laws are often the signature of hoping processes with a continuous distribution of trapping times [46].

Let us first examine the temperature behavior of $\beta$. The stretching exponent $\beta(T)$ decreases continuously
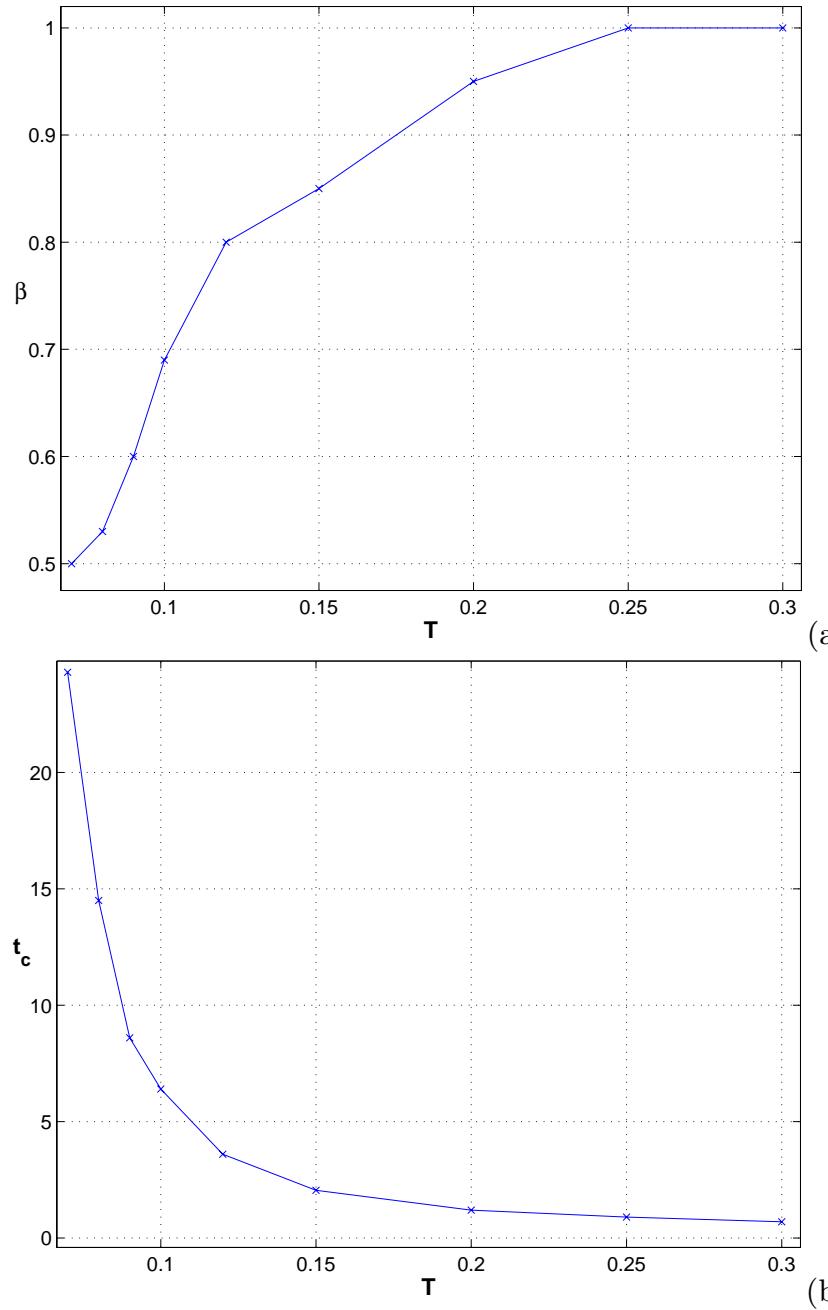

FIG. 10: Stretched exponential parameters in the steady state. (a) $\beta$, and (b) $t_{c}$ versus $T$.

from 1 at high temperature, to 0.5 at low temperature, as seen in Fig. 10a. We thus recover the classical exponential relaxation law for temperatures above $T \approx 0.25$, for lower temperatures the relaxation is anormal. The parameter $t_{c}(T)$, as shown in Fig. 10b, increases when the temperature is lowered. Below $T=0.07$, the aging effects become dominant, and we found that a fit of the contact correlation function similar to the one used for the quasi-equilibrium state (11), gives a very high value of the characteristic time $t_{c}\left(t_{w}, T\right) \approx 110$ (at $t_{w}=200$ and $T=0.05)$, showing the slow down of the relaxation in the globular state.

From Eq. (11) one may define a characteristic relaxation time $\tau$ ( $\tau$ and $t_{c}$ are of the same order of magnitude), using the relation,

$$
\tau \equiv \int_{0}^{\infty} P_{e q}(t) d t=\frac{t_{c}}{\beta} \Gamma(1 / \beta),
$$

here $\Gamma$ is the Euler gamma function. The characteristic relaxation time $\tau$ is shown in Fig. 11. It has an Arrhenius 


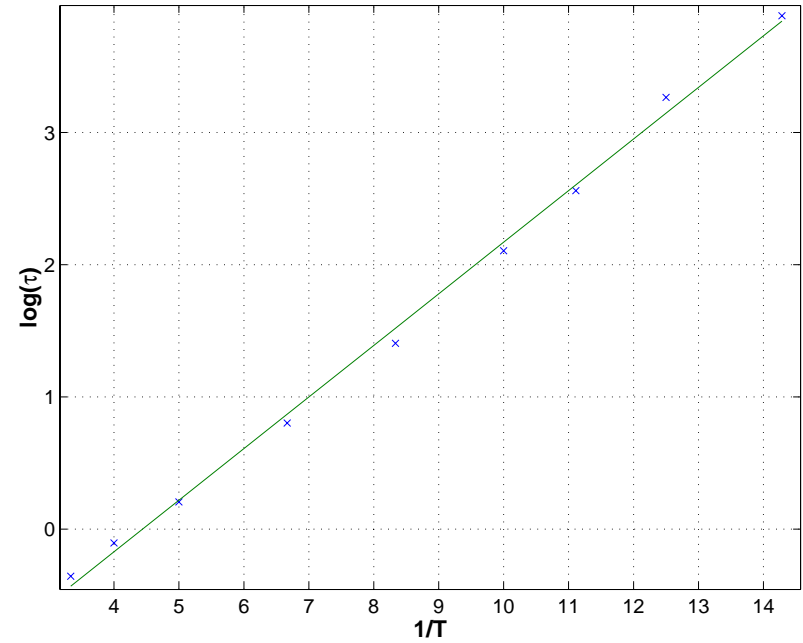

FIG. 11: Relaxation time as a function of the temperature: $\log (\tau)$ versus $1 / T$. The straight line corresponds to the Arrhenius law.

behavior,

$$
\tau=0.233 \exp \left(\frac{\Delta E}{T}\right)
$$

with an activation energy $\Delta E=0.21 \Theta$. This divergence of the relaxation time at low temperatures is characteristic of strong glasses [45]. It is well known that for polymer melts there exists a critical temperature $T_{c}$, related to the glassy transition, for which the relaxation time, as derived from the structure function, diverges algebraically $[47,48]$. In the case of single molecules, finite size effects are important, and no definite transition temperature was identified in our simulations. In the recent work [14] an extrapolation to lower temperatures was used to estimate the structure function relaxation time, and fitted with a power law using a very low value of $T_{c} \approx 0.03 \pm 0.01$ (their $\Theta$-point is higher than ours by a factor 3 , since their interaction potential is different). The principal manifestation of slowing down in the system was given by the change from exponential to stretched exponential relaxation. However, deviation from the Arrhenius law were not found for the relaxation time computed using the contact probability, as in formula (12). This may be related to the fact that the contact correlation function is an average over all monomers, irrespective of their spatial position (in this sense it is independent of $N$ for $N$ large enough). At variance, the structure function explicitly depends on the spatial distribution (through the wavevector). Therefore, the relaxation times deduced from the structure function are sensitive to the spatial inhomogeneities, and eventually to finite size effects.

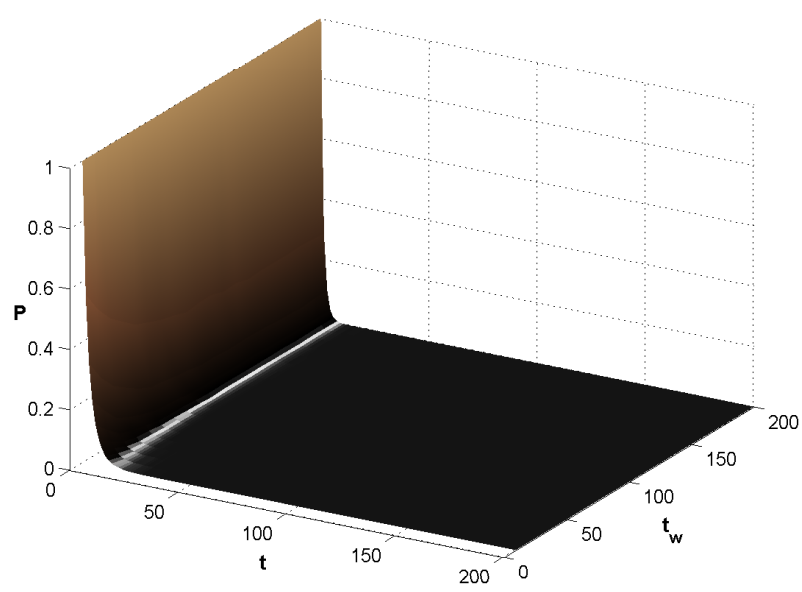

FIG. 12: Contact correlation function $P\left(t_{w}, t\right)$ for $T=0.05$ and $\lambda=0.01$.

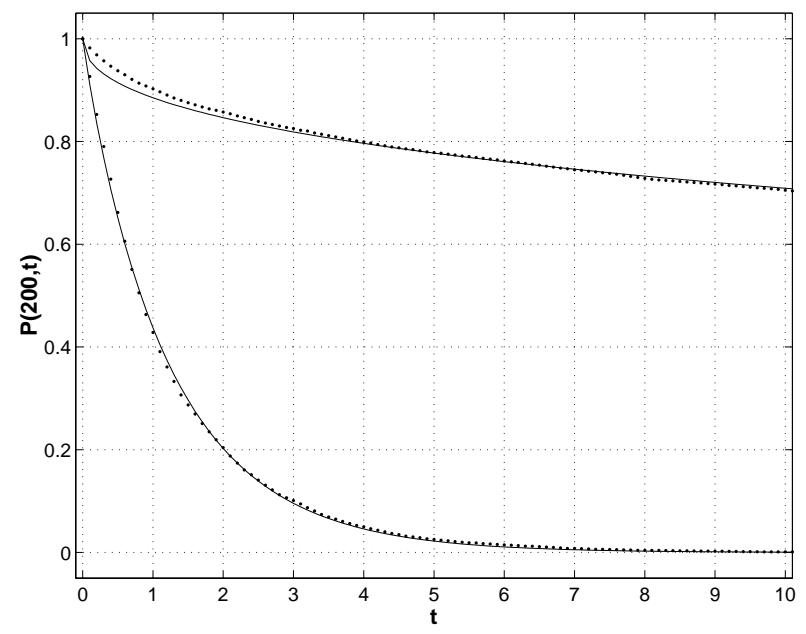

FIG. 13: The contact correlation function at $t_{w}=200$, solvent effects. Top: data for $T=0.05$ without solvent, the fit corresponds to a stretched exponential with $\beta=0.44$ and $t_{c}=110$. Bottom: same as top in the solvent case with $\lambda=0.01\left(\beta=0.95\right.$ and $\left.t_{c}=1.2\right)$.

\section{The effect of the solvent}

We also looked at the effect of the solvent on the contact correlation function (c.f. section IIB). We show in Fig. 12 a surface plot of $P\left(t_{w}, t\right)$ at a temperature of $T=0.05$ (it can be compared with Fig. 8), revealing that the relaxation is quite fast and that aging effects are negligible. A one-dimensional cut of this surface plot at $t_{w}=200$ is shown in Fig. 13 (together with a similar curve in the no solvent case), a time at which the globule is in quasi-equilibrium. The curve $P_{e q}(t)$, fitted as before by formula (11), shows that the relaxation law is now nearly exponential. We found $t_{c}=1.2$ and $\beta=0.94$. This should be contrasted with the results obtained at the same temperature without the effect of the solvent for which $t_{c}=110$ and $\beta=0.44$ (at comparable $t_{w}$ ). 


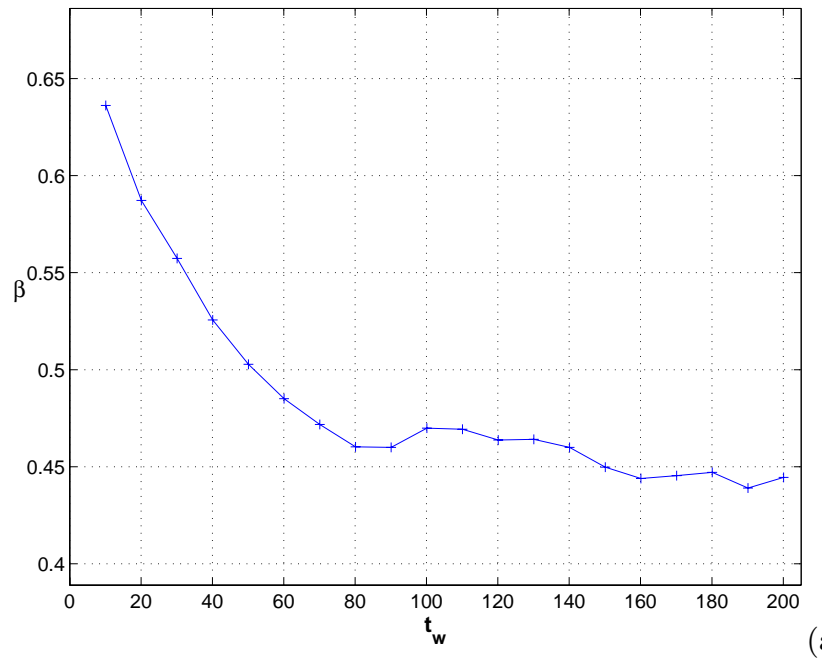

(a)

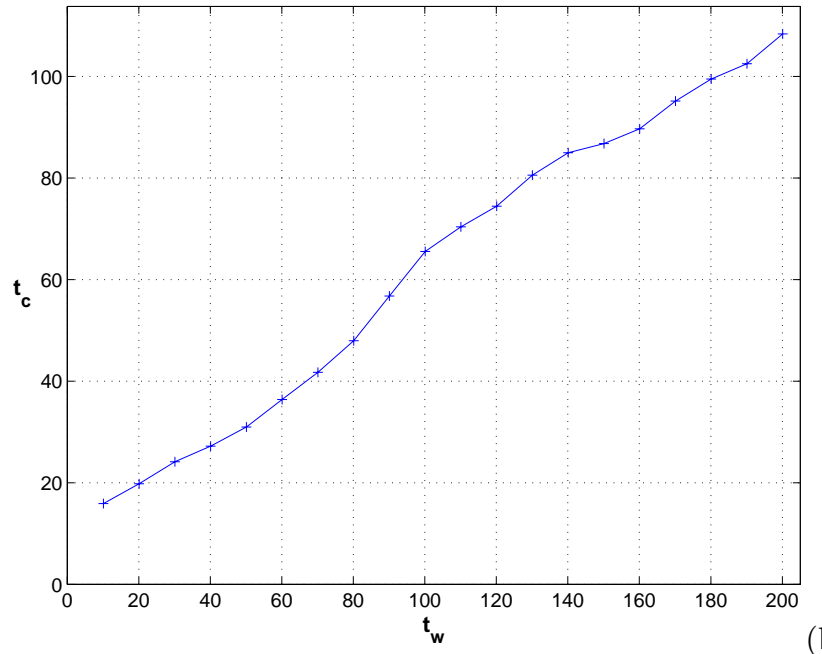

(b)

FIG. 14: Aging effects without solvent. (a) $\beta$ as a function of $t_{w}$ for $T=0.05$; (b) $t_{c}$ as a function of $t_{w}$ for $T=0.05$.

It is also interesting to compare these results with the evolution of the radius of gyration that was presented in figure 5. The collapse characteristic time scale increased in the presence of the solvent up to $t=150$, this is a factor 2 larger than without solvent. At variance, $t_{c}$ is strongly reduced, by a factor 50 . This means that, while the dynamics of collapse is slowed down (weaker effective attraction), the contact correlations are highly reduced as a consequence of the increased mobility of monomers (the effect of the solvent).

\section{The effect of aging}

Aging effects are characteristic of the time evolution of glassy systems after a temperature quench, like polymers melts, spin glasses or supercooled liquids [15]. They may reveal the non-equilibrium aspects of a system relaxing towards equilibrium. We thus studied the behavior of the contact correlation function as a function of the variable

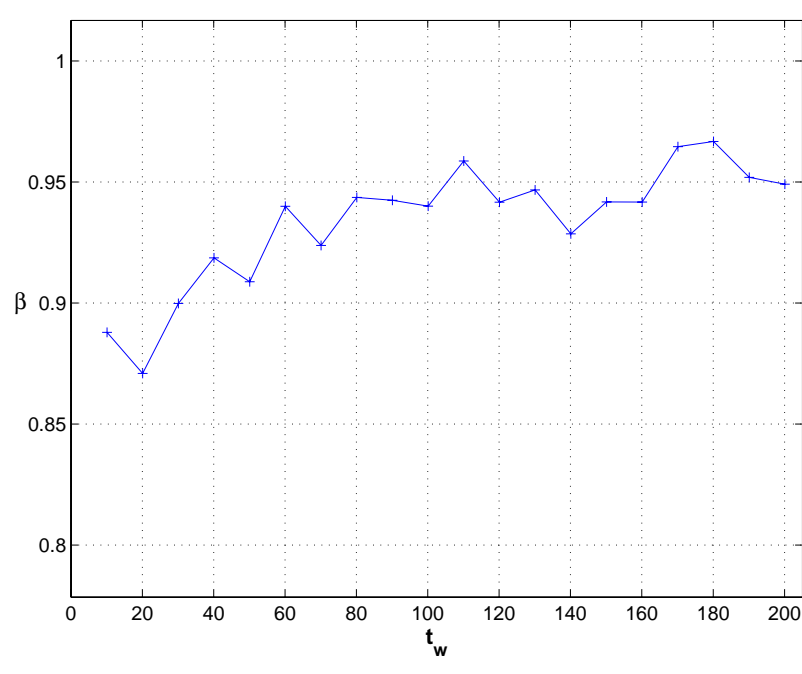

(a)

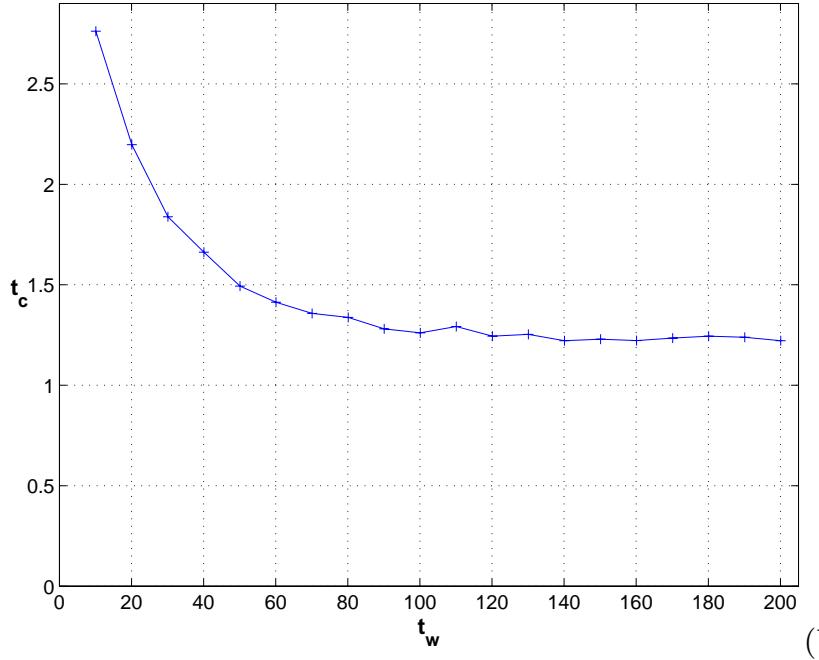

FIG. 15: Aging effects with solvent with $\lambda=0.01$. (a) $\beta$ as a function of $t_{w}$ for $T=0.05$; (b) $t_{c}$ as a function of $t_{w}$ for $T=0.05$.

$t_{w}$ at a fixed temperature $T=0.05$ with and without the solvent. Here again, we obtained a very good fit of $P\left(t_{w}, t\right)$ given by a stretched exponential function of the following form

$$
P\left(t_{w}, t\right)=\exp \left[-\left(\frac{t}{t_{c}\left(T, t_{w}\right)}\right)^{\beta\left(T, t_{w}\right)}\right],
$$

where the exponent $\beta$, which is positive and smaller than 1 , explicitly depends on $t_{w}$. This form has again only two free parameters $t_{c}$ and $\beta$ that both depend on $t_{w}$ and on $T$.

In the absence of solvent, the exponent $\beta$ decreases continuously as $t_{w}$ increases (Fig. 14a) and the characteristic time $t_{c}$ increases as $t_{w}$ increases (Fig. 14b). The dependence of the exponent $\beta$ on the waiting times $t_{w}$ might be related to the fact that the system has a complex energy landscape which is slowly organizing. This may gives rise to a stretched exponential behavior. The simple scaling form obtained in [14] for the dynamic structure 
factor is not verified in our case.

In the presence of solvent effects, the behavior of the characteristic time $t_{c}$ and the exponent $\beta$ completely change, as we can see comparing Fig. 14 with Fig. 15. The exponent $\beta$, which in the no solvent case differed significantly from unity, remained quasi-constant and nearly equal to one (Fig. 15a), showing that the relaxation is almost exponential, as in simple systems. We noted that $t_{c}$ first slightly decreases as $t_{w}$ increases, and then reach its asymptotic value (Fig. 15b). These properties are compatible with the reduction of monomer attraction in the globular state. The solvent effects render the globule fluid-like, eliminating the frustration which is at the origin of the glassy-like behavior.

\section{CONCLUSIONS}

We have studied the effect of the quench depth on the collapse of a homopolymer using molecular dynamics with the Nosé-Hoover thermostat. We have shown that depending on the quench depth, the polymer collapse following different pathways. For intermediate values of the quench depth, pearl like structures arising from initial density fluctuations are favored. For a strong quench, sausage like structures are privileged during the initial stage of the collapse, and the polymer is trapped in high energy meta-stable states. In fact, as the quench depth increases the frustration also increases and the globular state become more anisotropic, with freezed density inhomogeneities.

We have introduced an implicit solvent model in order to mimic the hydrophobic effect. In this model, the depth of the Lennard-Jones potential is modulated by the local density of monomers and no extra computational work is required. It contains one free parameter $\lambda$ allowing for a reduction of the monomer attraction; for large values of $\lambda$ monomer interactions become repulsive (in the condensed phase). We observed in the framework of this model that for strong quench, the polymer does not get stuck in a meta-stable state. It folds from the initial coil state, to a spherical molten globule, through a series of stages characterized by sausage-like structures. Even though these structures are similar to the ones encountered in the absence of solvent effects, the collapse relaxation is slower (as measured by the decay of the gyration radius), and the final state more homogenous. Most of solvent effects are related to the enhanced mobility of monomers, as the local density (the number of neighbors of a given monomer) increases.

We also studied quantitatively the collapse of the polymer by introducing a contact correlation function which exhibits non-classical relaxation behavior at low temperature. For inhomogeneous systems like a single polymer chain this quantity, based on the persistence of contacts between neighboring monomers, is more useful than the dynamic structure function (as applied to liquids or polymer melts). The contact correlation function can be fitted by a stretched exponential. We showed that the stretching exponent $\beta$ decreases when the temperature decreases and that the characteristic relaxation time $\tau$ displays classical Arrhenius behavior. We also found that aging effects are important at low temperatures (strong quench). The characteristic exponent $\beta$ and the relaxation time $\tau$, depend in a non trivial way on the waiting time, different to the self-similar law used in specific glassy systems [15].

The presence of a solvent strongly modifies the collapse process. Aging and anormal relaxation almost disappear. At very low temperatures we found that the characteristic exponent is slightly smaller than one. For such a low temperatures, in the absence of solvent, a steady state was not attained, and aging effects persisted. In contrast, the solvent permits a normal stationary relaxation process, and the system rapidly approaches an equilibrium state. The relaxation time, which diverges without solvent effects, becomes very short. A possible development of this work may be a comparison of the results obtained with our solvent model, with explicit solvent simulations.

The presence of stretched exponential relaxation in simple single homopolymer molecules, shows that a complex behavior already appears even in the absence of a diversity of monomer interactions or sequence effects specific to proteins. Therefore, it would be interesting to study the link between this complex dynamics and the reduction in the number of meta-stable states, characteristic of protein folding, through a self-organisation process driving the system to its native state.
[1] P. G. de Gennes, Scaling Concepts in Polymer Physics (Cornell, Ithaca, 1979).

[2] P. G. de Gennes, J. Phys. Lett. (Paris) 46, L639 (1985).

[3] B. Chu, Q. Ying, and A. Y. Grosberg, Macromolecules 28, 180 (1995).

[4] C. Wu and S. Zhou, Phys. Rev. Lett. 77, 3053 (1996).

[5] A. Y. Grosberg and A. R. Khokhlov, Statistical Physics of Macromolecules (AIP Press, New York, 1994).

[6] A. Y. Grosberg and A. R. Khokhlov, Giant Molecules (Academic press, New York, 1996).
[7] H. Frauenfelder, P. G. Wolynes, and R. H. Austin, Rev. Mod. Phys. 71, S419 (1999).

[8] A. Fersht, Structure and Mechanism in Protein Folding Science (Freeman, New York, 1999).

[9] C. Williams, F. Brochard, and H. L. Frisch, Ann. Rev. Phys. Chem. 32, 433 (1981).

[10] R. Chang and A. Yethiraj, J. Chem. Phys. 114, 7688 (2001).

[11] B. Haupt, T. Senden, and E. Sevick (2001), aPS, Seattle, www.aps.org/meet/MAR01/baps/abs/G2690009.html. 
[12] E. Pitard and J.-P. Bouchaud, Eur. Phys. J. E 5, 133 (2001).

[13] V. Rostiashvili, G. Migliorini, and T. A. Vilgis, Phys. Rev. E 64, 051112 (2001).

[14] N. V. Dokholyan, E. Pitard, S. V. Buldyrev, and H. E. Stanley, Phys. Rev. E 65, 030801R (2002).

[15] J.-P. Bouchaud, L. F. Cugliandolo, J. Kurchan, and M. Mézard, in Spin-Glasses and Random Fields, edited by A. Young (World Scientific, Singapore, 1997), condmat/9702070.

[16] S. Sastry, P. G. Debeneditti, and F. H. Stillinger, Nature 393, 554 (1998)

[17] A. Buguin, F. Brochard-Wyart, and P. G. de Gennes, C. R. Acad. Sci. Paris, Série 2 322, 741 (1996).

[18] A. Halperin and P. M. Goldbart, Phys. Rev. E 61, 565 (2000).

[19] B. Ostrovsky and Y. Bar-Yam, Europhys. Lett. 25, 409 (1994).

[20] B. Ostrovsky and Y. Bar-Yam, Biophys. Journ. 48, 1694 (1995).

[21] A. Pitard and H. Orland, Europhys. Lett. 41, 467 (1998).

[22] A. Byrne, P. Kiernan, D. Green, and K. A. Dawson, J. Chem. Phys. 1, 573 (1995).

[23] Y. A. Kuznetsov, E. G.Timoshenko, and K. A. Dawson, J. Chem. Phys. 103, 4807 (1995).

[24] Y. A. Kuznetsov, E. G. Timoshenko, and K. A. Dawson, J. Chem. Phys. 104, 3338 (1996).

[25] C. F. Abrams, N. Lee, and S. Obukhov (2001), preprint, http://xxx.lpthe.jussieu.fr/abs/cond-mat/0110491.

[26] N. Kikuchi, A. Gent, and J. M. Yeomans (2002), preprint, http://xxx.lpthe.jussieu.fr/abs/cond-mat/0201246.

[27] S. Takada, Z. Luthey-Schulten, and P. G. Wolynes, J. Chem. Phys. 110, 11616 (1999).

[28] T. Frisch and A. Verga, Phys. Rev. E 65, 041801 (2002).

[29] E. Rabani, J. D. Gezelter, and B. J. Berne, Phys. Rev Lett. 82, 3649 (1999).

[30] M. P. Allen and D. J. Tildesley, Computer Simulations of Liquids (Oxford, 2001).

[31] D. Frenkel and B. Smit, Understanding Molecular Simulations (Academic Press, 1996).

[32] A. Halperin and E. B. Zhulina, Macromolecules 24, 5393 (1991).

[33] A. Halperin and E. B. Zhulina, Europhys. Lett. 15, 417 (1991).

[34] P. Cifra and T. Bleha, Macro. Theory Simul. 4, 233 (1995).

[35] M. Wittkop, S. Kreitmeier, and D. Göritz, J. Chem. Phys 105, 3373 (1995).

[36] M. Wittkop, S. Kreitmeier, and D. Göritz, Phys. Rev. E 53, 838 (1996).

[37] P. Y. Lai, Phys. Rev. E 53, 3819 (1996).

[38] R. G. Maurice and C. C. Matthai, Phys. Rev. E 60, 3165 (1999).

[39] P. Grassberger and H.-P. Psu, Phys. Rev. E 65, 031807 (2002).

[40] E. G. Timoshenko, Y. A. Kuznetsov, and K. A. Dawson, J. Chem. Phys. 104, 3338 (1996).

[41] A. Milchev and K. Binder, Europhys. Lett 26, 671 (1994).

[42] W. Paul and M. Müller, J. Chem. Phys. 115, 630 (2001).

[43] Y. Zhou, C. Hall, and M. Karplus, Phys. Rev. Lett. 77, 2822 (1996).

[44] W. Gotze and L. Sjogren, Rep. Prog. Phys. 55, 241 (1992).

[45] P. G. Debenedetti, Metastable Liquids (Princeton University Press, Princeton, 1996).

[46] C. Monthus and J.-P. Bouchaud, J. Phys. A: Math. Gen. 29, 3847 (1996).

[47] K. Binder, J. Baschnagel, C. Bennemann, and W. Paul, J. Phys.: Condens. Matter 11, A47 (1999).

[48] J. Baschnagel, C. Bennemann, W. Paul, and K. Binder, J. Phys.: Condens. Matter 12, 6365 (2000). 